\title{
Neue Spitalfinanzierung ab 2012: Chance oder Katastrophe?
}

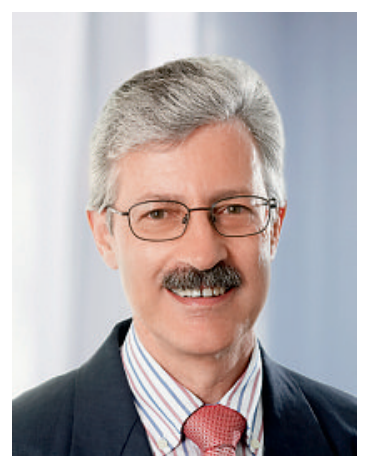

Die Einführung der neuen Spitalfinanzierung im Bereich der somatischen Akutversorgung durch Fallpauschalen nach SwissDRG wird schon bald Realität. Das Bevorstehen dieser bedeutenden Änderung löst verschiedene Reaktionen aus. Diese sind mit dem Schaudern vergleichbar, das bei einem Windstoss verspürt wird, der einen Wetterwechsel ankündigt, welcher schon lange zuvor vorhergesagt wurde, aber dessen man sich nicht bewusst war! Am 1. Januar 2012 wird der Systemwechsel endgültig Tatsache. Er wird eine ganze Reihe von neuen Mechanismen in Synergie bringen: Berücksichtigung der Investitionskosten und der Anlagenutzungskosten, freie Wahl des Behandlungsortes über die Kantonsgrenzen hinaus, Privatspitäler werden im Bereich der Grundversicherung den gleichen Regeln unterstellt usw. Dies wird eine grosse Zahl von Umstrukturierungen beschleunigen, die unabhängig vom System der Spitalfinanzierung jedenfalls unausweichlich geworden sind.

Unter diesen Voraussetzungen ist es nachvollziehbar, dass sich sowohl die Leistungserbringer als auch die Patientinnen und Patienten unter Umständen bedroht fühlen. Der vor kurzem lancierte Aufruf zu einem Moratorium entspricht der Ablehnung dessen, was im Grunde nur die Anwendung eines Gesetzes darstellt, das nach den demokratischen Regeln erlassen wurde. Das ist keine angemessene Reaktion auf die vorliegenden Probleme. Es geht im Gegenteil darum, das Potential einer solchen Dynamik zu nutzen, um im Bereich der Organisation und Struktur unserer Spitäler wahrnehmbare

\section{Es soll auf gesamtschweizerischer Ebene eine ein- heitliche Übergangsregelung beschlossen werden}

Verbesserungen zu fördern. In 16 Monaten werden sicherlich nicht alle Schwierigkeiten ausgeräumt sein. Es soll auf gesamtschweizerischer Ebene eine einheitliche Übergangsregelung beschlossen werden, um allen die Möglichkeit zu geben, sich mit dem neuen Fakturierungsinstrument vertraut zu machen, und um überdies während eines Zeitraums von zwei Jahren gewisse finanzielle Garantien beizubehalten. Gleichzeitig kann die SwissDRG AG während dieser Zeit die nachfolgenden Versionen der Tarifstruktur im Detail erarbeiten und dabei die Korrektur der beim Beginn festgestellten Ungenauigkeiten integrieren. Im Übrigen haben die meisten Kantone, die in den letzten Jahren die APDRG eingeführt haben, eine solche Übergangsphase angewandt.
Mit dem Grundsatz einer einheitlichen Spitalfinanzierung für die ganze Schweiz soll der Wettbewerb gefördert werden. Es ist eine interessante Tatsache, dass dieses Ziel vom Dachverband der Spitäler durchaus akzeptiert wird, während es bei den Kantonen vielfach Angstreflexe auslöst. Einige Kantone sind derzeit damit beschäftigt, sehr aktiv Gesetze zu erlassen, um die vollständige Kontrolle über die Leitung der Spitäler und deren Budgets beizubehalten. Dabei legen sie drakonische Kontrollbedingungen fest, damit die betreffenden Spitäler weiterhin auf der Spitalliste aufgeführt sind.

\section{Unter SwissDRG wird die massgebende Qualität dort erreicht, wo die ärzt- liche Kompetenz in einer passenden Infrastruktur über eine leistungsfähige Ausstattung verfügt}

Diesbezüglich scheint die unternehmerische Freiheit, auf welche die Mehrheit der eidgenössischen Räte so grossen Wert legt, recht begrenzt zu sein. Umgekehrt zeigen diese Anstrengungen auf kantonaler Ebene, dass auf dieser Stufe für die hängigen Probleme mehrere Lösungen gefunden werden können, insbesondere für die Finanzierung der Weiterbildung der Ärztinnen und Ärzte. Vor diesem Hintergrund ist es wichtig, unsere Behörden und unsere Kantonsparlamentarierinnen und -parlamentarier für diese Frage zu sensibilisieren, damit die entsprechenden Dossiers bis zum Ende des nächsten Jahres abgeschlossen werden können.

Ist die neue Spitalfinanzierung nun eine Chance oder eine Katastrophe? Eine Katastrophe kann nie ausgeschlossen werden, doch die Erfahrung der Kantone, die seit mehreren Jahren mit den DRG arbeiten, zeigt, dass bei ihnen keine negative Entwicklung zu verzeichnen war. Die freie Spitalwahl ist der Garant dafür, dass die therapeutische Freiheit nicht von der ökonomischen Logik verdrängt wird: Letztlich wird die Qualität massgebend sein. Diese kann nur von kompetenten Ärztinnen und Ärzten gewährleistet werden, die im Rahmen einer angemessenen Infrastruktur über eine leistungsfähige Ausstattung verfügen. Parallel dazu bieten diese Änderungen die Möglichkeit, Ordnung in die verschiedenen Prozesse zu bringen, den Einsatz der personellen und materiellen Ressourcen zu rationalisieren und zwischen dem medizinischen Personal und dem Verwaltungspersonal eine Kultur des Dialogs zu entwickeln. So gesehen ist die neue Spitalfinanzierung eine Chance. Wir müssen nun entscheiden, ob wir diese Chance zum Wohl unserer Patientinnen und Patienten und im Hinblick auf eine positive Beeinflussung unseres Arbeitsumfelds ergreifen wollen.

Dr. P.-F. Cuénoud

Mitglied des Zentralvorstands der FMH Verantwortlicher Ressort SwissDRG 